\title{
Deformable Atlas for Multi-structure Segmentation
}

\author{
Xiaofeng Liu^, Albert Montillo, Ek. T. Tan, John F. Schenck, and Paulo \\ Mendonca \\ General Electric Global Research Center, Niskayuna, NY USA \\ xiaofeng.liu@ge.com
}

\begin{abstract}
We develop a novel deformable atlas method for multistructure segmentation that seamlessly combines the advantages of image-based and atlas-based methods. The method formulates a probabilistic framework that combines prior anatomical knowledge with imagebased cues that are specific to the subject's anatomy, and solves it using expectation-maximization method. It improves the segmentation over conventional label fusion methods especially around the structure boundaries, and is robust to large anatomical variation. The proposed method was applied to segment multiple structures in both normal and diseased brains and was shown to significantly improve results especially in diseased brains.
\end{abstract}

Keywords: Segmentation, deformable atlas, label fusion, MLE, GVF.

\section{Introduction}

Segmenting multiple structures from medical images remains a difficult task due to the large variability of structure shape, their appearance in images, and the lack of contrast between neighboring structures. One can roughly divide existing segmentation methods into two categories: image-based approaches and atlasbased approaches.

Image-based approaches are based on image cues, e.g., intensity, gradient, texture. Among them deformable models, i.e., active contour 1] and level set methods 2], have been widely adopted and shown success on many applications. Atlas-based approaches [3, 4] rely largely on the prior knowledge about the spatial arrangement of structures. They are generally performed by first registering atlas images to the subject image, called target, so that the manual segmentations on the atlases are propagated and fused to segment the target. Compared to image-based approaches, these methods incorporate prior anatomical knowledge, but they do not explicitly consider images cues and thus are limited by large anatomical variation and imperfect registration. Recently methods were developed to incorporate image information into atlas-based approach [5 7], but image cues other than intensity were not exploited. To the best of our knowledge,

^ Corresponding author.

K. Mori et al. (Eds.): MICCAI 2013, Part I, LNCS 8149, pp. 743-750, 2013.

(C) Springer-Verlag Berlin Heidelberg 2013 


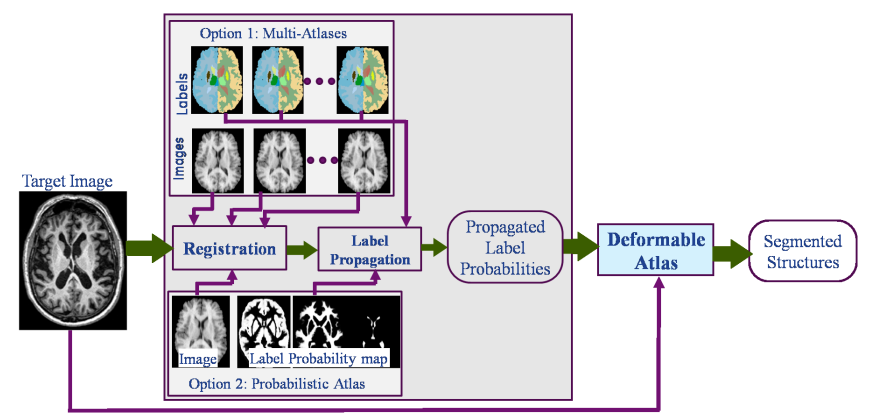

Fig. 1. Multi-structure segmentation using deformable atlas approach

the only work that attempted to close the gap between image-based and atlasbased methods is spectral label fusion [8]. However the method is region-based and it is difficult to extend to multiple structures especially when the boundaries between neighboring structures are weak.

In this paper we develop a novel multi-structure segmentation method, called deformable atlas, that seamlessly combines the advantages of image-based and atlas-based methods. The method formulates and solves a probabilistic framework that incorporates prior anatomical knowledge with image cues that are specific to the target images, including structure intensity profiles and boundaries. Significant improvements were demonstrated over common label fusion methods on the multi-structure segmentation of both normal and diseased brains.

\section{Methods}

Fig. 1 shows the flowchart of multi-structure segmentation using deformable atlas (DA) method. DA accepts as inputs the target image and spatial priors about the structures. The spatial priors can be generated using either a multiatlas approach or a spatial probabilistic atlas. After that, DA segments the multi structures simultaneously. Consider a target image $\mathbf{I}$, where $I_{j}$ is the intensity at voxel $j$ with $j \in\{1,2, \ldots, J\}$. Let $K$ be the number of structures or labels. The true label is represented by $\mathbf{z}_{j}=\left[z_{j 1}, \ldots, z_{j K}\right]$, where $z_{j k}=1$ if $j$ belongs to structure $k$, and 0 otherwise. The label spatial prior is $f\left(\mathbf{z}_{j}\right)=\mathbf{p}_{j}=\left[p_{j 1}, \ldots, p_{j K}\right]$. In a multi-atlas approach, $p_{j k}=\frac{1}{N} \sum_{n=1}^{N} L_{j k}^{n}$ with $L_{j k}^{n}$ being the propagated label at $j$ from the $n^{\text {th }}$ atlas and $N$ being the number of atlases. In a probabilistic atlas approach, $\mathbf{p}_{j}$ is the propagated spatial prior after registration. Let $\boldsymbol{\theta}=$ $\{\boldsymbol{\rho}, \boldsymbol{\pi}\}$ be the set of unknown parameters, where $\boldsymbol{\rho}=\left\{\rho_{1}, \ldots \rho_{K}\right\}$ are the intensity distribution functions for the $K$ structures, and $\boldsymbol{\pi}=\left\{\pi_{j k}\right\}$ with $\pi_{j k}$ being the probability that voxel $j$ belongs to structure $k$ and $\sum_{k=1}^{K} \pi_{j k}=1$ for all $j$.

The deformable atlas method employs a maximum likelihood estimation (MLE) framework that combines label spatial prior knowledge with image-based cues, i.e., intensities and edges to improve the multi-structure segmentation. Using Bayes' law, the likelihood function is expressed as $f(\mathbf{Z}, \mathbf{I} \mid \boldsymbol{\theta})=$

$$
f(\mathbf{I} \mid \mathbf{Z}, \boldsymbol{\theta}) f(\mathbf{Z} \mid \boldsymbol{\theta}) \propto f(\mathbf{I} \mid \mathbf{Z}, \boldsymbol{\rho}) f(\mathbf{I} \mid \mathbf{Z}, \boldsymbol{\pi}) f(\mathbf{Z} \mid \boldsymbol{\theta}) \propto f(\mathbf{I} \mid \mathbf{Z}, \boldsymbol{\rho}) f(\boldsymbol{\pi} \mid \mathbf{I}) f(\mathbf{Z} \mid \boldsymbol{\pi})
$$




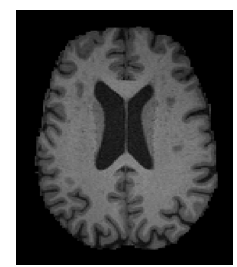

(a)

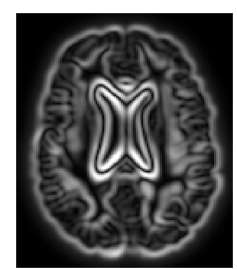

(b)

Fig. 2. Example of the speed function. (a) A skull-striped brain T1 MR image, and (b) the magnitude of GVF.

with the assumptions that $\boldsymbol{\rho}$ and $\boldsymbol{\pi}$ are independent, $\mathbf{Z}$ and $\boldsymbol{\rho}$ are independent, and $\mathbf{I}$ and $\mathbf{Z}$ are conditionally independent given $\boldsymbol{\pi}$. The term $f(\mathbf{I} \mid \mathbf{Z}, \boldsymbol{\rho})$ is based on the structure intensity profiles in the target image, while $f(\boldsymbol{\pi} \mid \mathbf{I})$ models the distribution of $\boldsymbol{\pi}$ given $\mathbf{I}$ and is defined based on structure boundaries (described below). We then develop an Expectation-Maximization(EM) algorithm to solve for $\boldsymbol{\theta}$, which iterates between the E-step and the M-step.

\subsection{The Probability $f(\pi \mid \mathrm{I})$}

While most label fusion approaches do not explicitly explore structure boundary information, image-based methods have shown that structure boundaries are crucial for accurate segmentation. Here we conceptually define the log probability as

$$
\log f(\boldsymbol{\pi} \mid \mathbf{I})=\log C e^{-\gamma \sum_{k=1}^{K} F_{\mathbf{I}}\left(\mathbf{S}_{\mathbf{k}}\right)}=\log C-\gamma \sum_{k=1}^{K} F_{\mathbf{I}}\left(\mathbf{S}_{\mathbf{k}}\right),
$$

where $F_{\mathbf{I}}\left(\mathbf{S}_{k}\right)$ is a potential energy function defined on the boundaries $\mathbf{S}_{k}$ of the $k^{\text {th }}$ structure segmented based on $\boldsymbol{\pi}$, which typically takes local minimum at edges along structure contours. $C$ is a normalization constant.

In classic deformable models $F_{\mathbf{I}}\left(\mathbf{S}_{k}\right)$ often does not have an analytical form and minimizing it does not lead to a closed-form solution. Instead, it is optimized iteratively by either guiding the contour deformation using force fields in active contours [1], or evolving the level set function using speed functions in level set methods [9]. Inspired by that, we define pseudo level set functions $\phi_{j k}=$ $\pi_{j k}-\sum_{i \neq k} \pi_{j i}$, which are similar to standard level set functions except their values are constrained to $[-1,1] . \phi_{j k} \in(0,1]$ when $i$ is inside structure $k$, and $\phi_{j k} \in[-1,0)$ if $i$ is outside structure $k$. As in level set methods, the evolution of $\phi(j)$ to maximize Eqn. (2) can be expressed as $\phi_{j k}^{s+1}-\phi_{j k}^{s}=-\gamma \mathbf{v}_{j} \cdot \nabla \phi_{j k}$, with $\mathbf{v}_{j}$ being a speed function, and $s$ being the evolution step. It is equivalent to

$$
\pi_{j k}^{s+1}-\pi_{j k}^{s}=-\gamma \mathbf{v}_{j} \cdot \nabla \pi_{k j}
$$

under the condition that $\sum_{k=1}^{K} \pi_{j k}=1$. We use the gradient vector flow (GVF) [10] as the speed function, because it has been shown to be more flexible and provide stronger constraints than many other forces or speed functions [10]. 
Here we compute it from the magnitude of target image gradient $\left\|\nabla_{\mathbf{x}} \mathbf{I}\right\|$ instead of binary edge map, i.e., $\mathbf{v}_{j}=G V F\left\{\nabla\left\|\nabla_{\mathbf{x}} I_{j}\right\|\right\}$ An example is shown in Fig. 2 .

A notable advantage of this formulation is $\pi_{j k}$ only evolves in regions where their labels are ambiguous based on spatial priors. For regions with definite labels, the term $\nabla \pi_{k j}$ in Eqn. (3) equals 0 and thus $\pi_{j k}$ does not evolve.

\subsection{The E-Step}

In the E-step, the conditional expectation of the log likelihood function is computed. Let $\boldsymbol{\theta}^{(t)}$ be the set of estimated parameters at iteration $t$. As in standard $\mathrm{EM}$ algorithm, the conditional expectation is

$$
\begin{aligned}
Q\left(\boldsymbol{\theta} \mid \boldsymbol{\theta}^{(t)}\right) & =E\{\log f(\mathbf{I} \mid \mathbf{Z}, \boldsymbol{\rho}) f(\mathbf{Z} \mid \boldsymbol{\pi})\}+\log f(\boldsymbol{\pi} \mid \mathbf{I}) \\
& =\sum_{\mathbf{Z}}[\log f(\mathbf{I} \mid \mathbf{Z}, \boldsymbol{\rho}) f(\mathbf{Z} \mid \boldsymbol{\pi})] f\left(\mathbf{Z} \mid \mathbf{I}, \boldsymbol{\theta}^{(t)}\right)+\log f(\boldsymbol{\pi} \mid \mathbf{I})
\end{aligned}
$$

Using Bayes' law and assuming the labels and intensities at voxels are independently distributed we have

$$
f\left(\mathbf{Z} \mid \mathbf{I}, \boldsymbol{\theta}^{(t)}\right)=\frac{f\left(\mathbf{I} \mid \mathbf{Z}, \boldsymbol{\theta}^{(t)}\right) f(\mathbf{Z})}{\sum_{\mathbf{Z}^{\prime}} f\left(\mathbf{I} \mid \mathbf{Z}^{\prime}, \boldsymbol{\theta}^{(t)}\right) f\left(\mathbf{Z}^{\prime}\right)}=\frac{\prod_{j} \prod_{k}\left[f\left(I_{j} \mid z_{j k}, \boldsymbol{\theta}^{(t)}\right) p_{j k}\right]^{z_{j k}}}{\sum_{\mathbf{Z}^{\prime}} \prod_{j} \prod_{k}\left[f\left(I_{j} \mid z_{j k}^{\prime}, \boldsymbol{\theta}^{(t)}\right) p_{j k}\right]^{z_{j k}^{\prime}}} .
$$

Thus at each voxel $j$ we have

$$
w_{j k}^{(t)}=f\left(z_{j k}=1 \mid I, \boldsymbol{\theta}^{(t)}\right)=\frac{f\left(I_{j} \mid z_{j k}=1, \boldsymbol{\theta}^{(t)}\right) p_{j k}}{\sum_{k^{\prime}=1}^{K} f\left(I_{j} \mid z_{j k^{\prime}}=1, \boldsymbol{\theta}^{(t)}\right) p_{j k^{\prime}}},
$$

and $w_{j k}$ is referred as the weighting variable. $\rho_{k}^{(t)}\left(I_{j}\right)=f\left(I_{j} \mid z_{j k}=1, \boldsymbol{\theta}^{(t)}\right)$ is the intensity distribution for structure $k$. Eqn. (4) can be expressed as

$$
\begin{aligned}
Q\left(\boldsymbol{\theta} \mid \boldsymbol{\theta}^{(t)}\right) & =\sum_{j} \sum_{k}\left[\log f\left(I_{j} \mid z_{j k}=1, \rho_{k}\right) f\left(z_{j k}=1 \mid \pi_{j k}\right)\right] w_{j k}^{(t)}+\log f(\boldsymbol{\pi} \mid \mathbf{I}) \\
& =\sum_{j} \sum_{k} w_{j k}^{(t)} \log \rho_{k}\left(I_{j}\right)+\sum_{j} \sum_{k} w_{j k}^{(t)} \log \pi_{j k}+\log f(\boldsymbol{\pi} \mid \mathbf{I}) .
\end{aligned}
$$

\subsection{The M-Step}

In the M-step, the parameters $\boldsymbol{\theta}^{(t+1)}$ are computed by maximizing $Q\left(\boldsymbol{\theta} \mid \boldsymbol{\theta}^{(t)}\right)$.

To estimate $\boldsymbol{\rho}$, we model the intensity distribution using Parzen window method, i.e., $\rho_{k}(x)=\sum_{j} a_{k j} G\left(x ; I_{j}, \sigma\right)$, where $G\left(\cdot ; I_{j}, \sigma\right)$ is the Gaussian kernel with mean $I_{m}$ and standard deviation $\sigma . a_{k j}$ are the coefficients such that $\sum_{j} a_{k j}=1$. By maximizing Eqn. (7) it is derived that

$$
\rho_{k}^{(t+1)}=\arg \max _{\rho_{k}} \sum_{j} w_{j k}^{(t)} \log \rho_{k}\left(I_{j}\right)=\frac{1}{\sum_{j^{\prime}} w_{j^{\prime} k}^{(t)}} \sum_{j} w_{j k}^{(t)} G\left(x ; I_{j}, \sigma\right),
$$

or $a_{k j}^{(t+1)}=w_{j k}^{(t)} / \sum_{j^{\prime} k} w_{j^{\prime} k}^{(t)}$. 


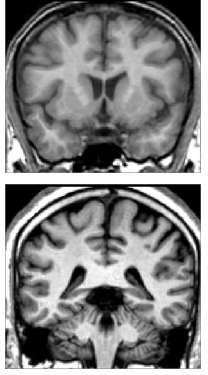

(a)

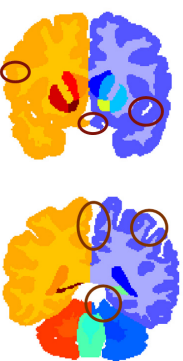

(b)

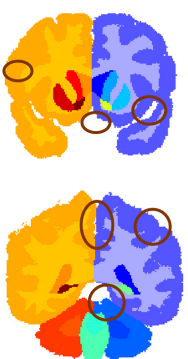

(c)

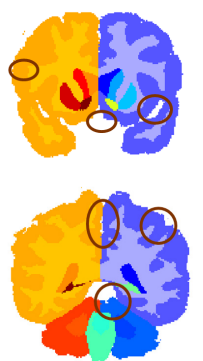

(d)
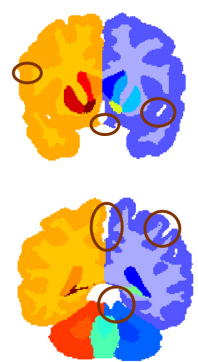

(e)

Fig. 3. Results on two IBSR subjects. (a) T1-weighted coronal slices, (b) ground truth, the results of (c) MV, (d) IWV, and (e) DA methods. The improvements of DA are highlighted using circles.

To etimate $\boldsymbol{\pi}$, based on Eqn. (7) we have

$$
\boldsymbol{\pi}^{(t+1)}=\arg \max _{\boldsymbol{\pi}} \sum_{j} \sum_{k} w_{j k}^{(t)} \log \pi_{j k}+\log f(\boldsymbol{\pi} \mid \mathbf{I})
$$

with the constraints $\sum_{k} \pi_{j k}=1$ for all $j$, or $c_{j}\left(\pi_{j 1}, \ldots, \pi_{j K}\right)=1-\sum_{k} \pi_{j k}=0$. As defined earlier, the term $\log f(\boldsymbol{\pi} \mid \mathbf{I})$ does not have an analytical form and thus Eqn. (9) does not have a closed-form solution. Instead we solve it iteratively using extended gradient descent method [11]. Let $\boldsymbol{\pi}_{j}=\left[\pi_{j 1}, \ldots, \pi_{j K}\right]^{T}$, and we denote the gradient as $\mathbf{g}\left(\boldsymbol{\pi}_{j}\right)=\nabla_{\boldsymbol{\pi}_{j}} Q\left(\boldsymbol{\theta} \mid \boldsymbol{\theta}^{(t)}\right)=\left[g\left(\pi_{j 1}\right), \ldots, g\left(\pi_{j K}\right)\right]^{T}$, such that

$$
g\left(\pi_{j k}\right)=\frac{\partial Q\left(\boldsymbol{\theta} \mid \boldsymbol{\theta}^{(t)}\right)}{\partial \pi_{j k}}=\frac{w_{j k}^{(t)}}{\pi_{j k}}-\gamma \mathbf{v}_{j} \cdot \nabla \pi_{k j} .
$$

Because of the constraints, $g\left(\pi_{j k}\right)$ needs to be projected onto the constrained space [11], i.e., $\mathbf{g}_{N}\left(\boldsymbol{\pi}_{k}\right)=\mathbf{g}\left(\boldsymbol{\pi}_{k}\right)-\frac{\nabla c_{j} \cdot \mathbf{g}\left(\boldsymbol{\pi}_{k}\right)}{\left\|\mathbf{g}\left(\boldsymbol{\pi}_{k}\right)\right\|^{2}} \nabla c_{j}$, or equivalently,

$$
g_{N}\left(\pi_{j k}\right)=g\left(\pi_{j k}\right)-\sum_{k=1}^{K} g\left(\pi_{j k}\right) / K
$$

At iteration $s, \pi_{j k}^{(t+1) s+1}$ is updated as

$$
\pi_{j k}^{(t+1) s+1}-\pi_{j k}^{(t+1) s}=\delta g_{N}\left(\pi_{j k}^{(t+1) s}\right),
$$

where $\delta$ is the small step size, and $\pi_{j k}^{(t+1) 0}=\pi_{j k}^{(t)}$. After that $\pi_{j k}^{(t+1) s+1}$ is normalized to satisfy the constraint that $\sum_{k} \pi_{j k}^{(t+1) s+1}=1$.

The complete deformable atlas algorithm is summarized in Algorithm 1

\section{Experiments and Results}

Experiments were first performed using the Internet Brain Segmentation Repository (IBSR) data set11. It contains 18 healthy subjects with $\mathrm{T} 1$ weighted images,

${ }^{1}$ Provided by the Center for Morphometric Analysis at Massachusetts General Hospital and available at http://www.cma.mgh.harvard.edu/ibsr/ 
Data: Target image $\mathbf{I}$, prior spatial probability $\mathbf{p}_{j}$

Initialization: Set maximum iterations $T$ and $S$, set $t=0, w_{j k}^{(0)}=\pi_{j k}^{(0)}=p_{j k}$; Compute $\mathbf{v}_{j}$ using GVF and $\rho_{k}^{(0)}$ using Eqn. (8) ; repeat

The $\boldsymbol{E}$-Step: compute $w_{j k}^{(t)}$ as in Eqn. (6);

The M-Step: compute $\rho_{k}^{(t+1)}$ using (8), set $\mathrm{s}=0, \pi_{j k}^{(t+1) 0}=w_{j k}^{(t)}$; repeat

Compute $\pi_{j k}^{(t+1) s+1}$ using Eqn. (12);

Normalize $\pi_{j k}^{(t+1) s+1}=\pi_{j k}^{(t+1) s+1} / \sum_{k^{\prime}=1}^{K} \pi_{j k^{\prime}}^{(t+1) s+1}$;

$\mathrm{s}=\mathrm{s}+1$;

until it converges or $s>S$;

$z_{j k}=1$ if $\pi_{j k}^{(t+1)}>\pi_{j i}^{(t+1)}$ for all $i \neq k$, otherwise $z_{j k}=0$;

$\mathrm{t}=\mathrm{t}+1$;

until the algorithm converges or $t>T$;

Algorithm 1. The deformable atlas algorithm

and 32 brain structures were manually delineated on each image by experts. We also tested on Alzheimer's disease brains using the Australian Imaging, Biomarkers and Lifestyle (AIBL) data sets. For comparison, experiments were performed using three methods: majority voting (MV), intensity weighted voting (IWV), and DA. For MV, the segmentation was determined by fusing propagated label maps without considering image cues [3], i.e., j was labeled as $k$ if $p_{j k}>p_{j i}$ for $\forall i \neq k$. IWV improves MV by considering structure-specific intensity profiles, i.e., the intensity weighting $f(\mathbf{I} \mid \mathbf{Z}, \rho)$ in Eqn. (1) was applied but the term for structure boundary $f(\boldsymbol{\pi} \mid \mathbf{I})$ was ignored. For DA, both the intensity weighting and the structure boundary term were applied. The parameters were empirically selected: $\gamma=0.5$ and $\delta=0.05 . \sigma$ in Eqn. (8) was chosen as the intensity standard deviation of all voxels in each structure. In all experiments, the image registration was performed using $\mathrm{SyN}$ method [12].

For IBSR data, 18 leave-one-out experiments were performed using a multiatlas approach. The segmentation results were compared to the manual segmentation and evaluated using the Dice coefficient, i.e., $D=\frac{2|X \cap Y|}{|X \cup Y|}$ where $X$ and $y$ are the voxel sets of manual labeling and automated segmentation result, respectively, and $|\cdot|$ is the set cardinality. Fig. 3] shows the qualitative results on two data sets, and Fig. 4 shows the quantitative results for all structures. Left and right structures are combined for clarity, and results on vessels were not shown. It was observed that IWV performed much better than MV in most structures, which demonstrated the effectiveness of incorporating intensity into the voting strategy. DA further improved the results especially in the ventricles and the cortex, and worked sightly better or similarly on other structures. The DA results are comparable to or better than state-of-the-art brain segmentation algorithms as shown in [13].

We also tested the methods on 45 AIBL images on Alzheimer's disease using the multi-atlas approach with the 18 IBSR data as the atlases, and the results were visually inspected. Fig. 5 shows the results on three selected subjects. 


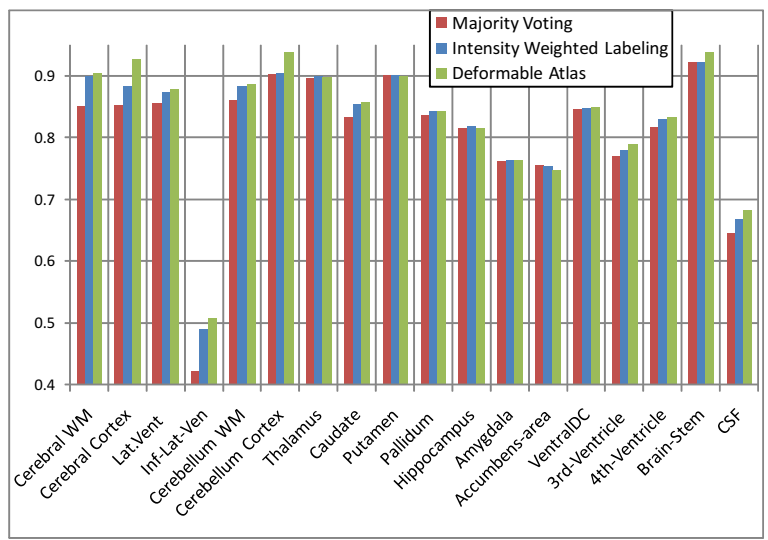

Fig. 4. The mean Dice coefficients of the three methods on different brain structures
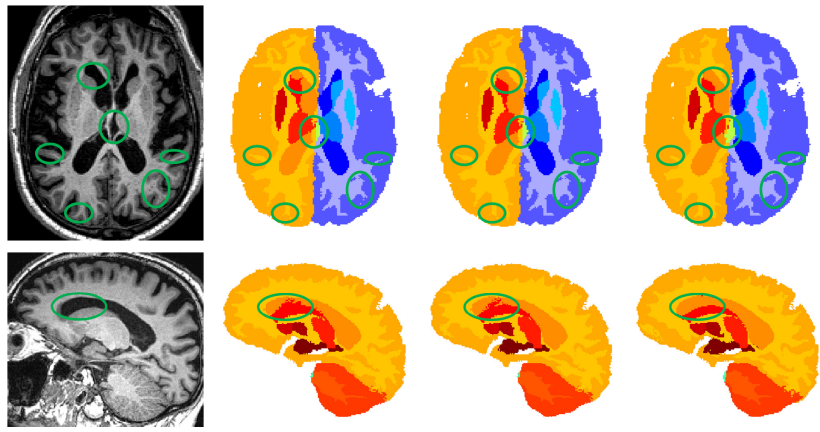

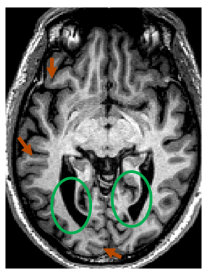

(a)

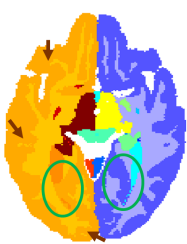

(b)

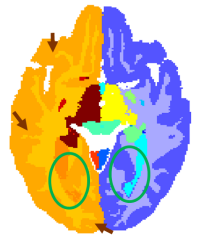

(c)

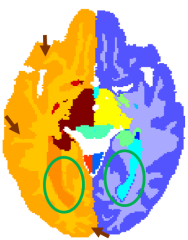

(d)

Fig. 5. Results on three AIBL images. (a) The T1-weighted images, the results of (b) MV, (c) IWV, and (d) DA methods. The major differences are highlighted using circles and arrows.

It was found that DA consistently worked much better than MV and IWV. This was because the diseased brains have large anatomical changes as compared to normal brains, e.g., brain tissue shrinkage and ventricle enlargement. Since the atlas images were all on normal brains, these pathological differences were not captured by the registration algorithm and resulted in failure of MV and IWV on certain parts of the brain (circled regions in Fig. 5). DA worked very well despite the anatomical changes thanks to the edge-based deformation. These results showed that deformable atlas could be successfully applied to brains with large deformation that could not be handled by voting based methods. 


\section{Discussions}

We developed a deformable atlas method for multi-structure segmentation that combines the benefits of atlas-based and image-based approaches, and applied it to segment both normal brains and brains with Alzheimer's disease. Results showed that the method performed very well especially for diseased brain in spite of large anatomical deformation while other segmentation methods failed. The method can be readily extended to other applications of atlas-based segmentation, e.g., prostate and heart. Though only demonstrated using a multi-atlas approach, the method can equally be applied to probabilistic atlas approaches. Part of our future work is to include other speed functions, including curvaturebased terms for smoothness, to get better segmentation results.

\section{References}

1. Kass, M., Witkin, A., Terzopoulos, D.: Snakes: Active contour models. Int. J. Comp. Vis. 1(4), 321-331 (1988)

2. Malladi, R., Sethian, J.A., Vemuri, B.C.: Shape modeling with front propagation: a level set approach. IEEE Trans. Patt. Anal. Mach. Intell. 17(2), 158-175 (1995)

3. Heckemann, R., Hajnal, J., Aljabar, P., Rueckert, D., Hammers, A.: Automatic anatomical brain MRI segmentation combining label propagation and decision fusion. Neuroimage 33(1), 115-126 (2006)

4. Warfield, S., Zou, K., Wells, W.: Simultaneous truth and performance level estimation (STAPLE): An algorithm for the validation of image segmentation. IEEE Trans. Med. Imag. 23(7), 903-930 (2004)

5. Artaechevarria, X., Munoz-Barrutia, A., de Solorzano, C.O.: Combination strategies in multi-atlas image segmentation: Application to brain MR data. IEEE Trans. Med. Imag. 28(8), 1266-1277 (2009)

6. Sabuncu, M.R., Yeo, B.T.T., Leemput, K.V., Fischl, B., Golland, P.: A generative model for image segmentation based on label fusion. IEEE Trans. Med. Imag. 29(10), 1714-1729 (2010)

7. Shiee, N., Bazin, P.L., Cuzzocreo, J.L., Blitz, A., Pham, D.L.: Segmentation of brain images using adaptive atlases with application to ventriculomegaly. Inf. Process. Med. Imag., 1-22 (2011)

8. Wachinger, C., Golland, P.: Spectral label fusion. In: Ayache, N., Delingette, H., Golland, P., Mori, K. (eds.) MICCAI 2012, Part III. LNCS, vol. 7512, pp. 410-417. Springer, Heidelberg (2012)

9. Xu, C., Yezzi, A., Prince, J.: A summary of geometric level-set analogues for a general class of parametric active contour and surface models. In: Workshop on Variational and Level Set Methods in Computer Vision, pp. 104-111 (2001)

10. Xu, C., Pham, D.L., Prince, J.L.: Medical Image Segmentation Using Deformable Models. In: SPIE Handbook on Medical Imaging. Medical Image Analysis, vol. III, SPIE (2000)

11. Rosen, J.B.: The gradient projection method for nonlinear programming: Part II, nonlinear constraints. J. Soc. Indust. Appl. Math. 9(4), 514-532 (1961)

12. Avants, B.B., Epstein, C.L., Grossman, M., Gee, J.C.: Symmetric diffeomorphic image registration with cross-correlation: Evaluating automated labeling of elderly and neurodegenerative brain. Med. Image Anal. 12(1), 26-41 (2008)

13. Roussau, F., Habas, P., Studholme, C.: A supervised patched-based approach for human brain labeling. IEEE Trans. Med. Imag. 30(10), 1852-1862 (2011) 